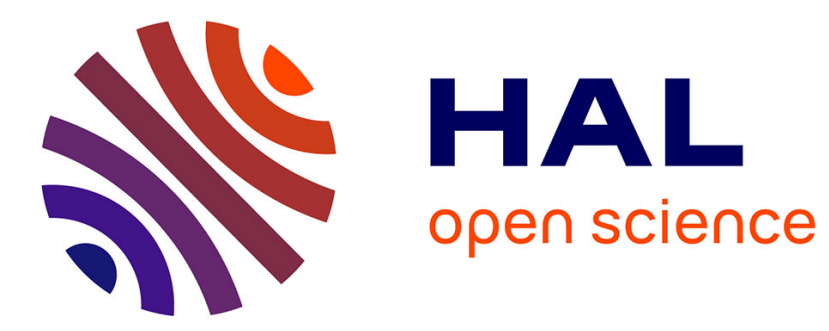

\title{
On the sensitivity analysis of porous material models
}

Morvan Ouisse, Mohamed Ichchou, Slaheddine Chedly, Manuel Collet

\section{To cite this version:}

Morvan Ouisse, Mohamed Ichchou, Slaheddine Chedly, Manuel Collet. On the sensitivity analysis of porous material models. Journal of Sound and Vibration, 2012, 331 (24), pp.5292-5308. 10.1016/j.jsv.2012.07.018 . hal-02300228

\section{HAL Id: hal-02300228 \\ https://hal.science/hal-02300228}

Submitted on 20 Oct 2020

HAL is a multi-disciplinary open access archive for the deposit and dissemination of scientific research documents, whether they are published or not. The documents may come from teaching and research institutions in France or abroad, or from public or private research centers.
L'archive ouverte pluridisciplinaire HAL, est destinée au dépôt et à la diffusion de documents scientifiques de niveau recherche, publiés ou non, émanant des établissements d'enseignement et de recherche français ou étrangers, des laboratoires publics ou privés. 


\title{
On the sensitivity analysis of porous material models
}

\author{
Morvan Ouisse $^{\mathrm{a}, *}$, Mohamed Ichchou ${ }^{\mathrm{b}}$, Slaheddine Chedly ${ }^{\mathrm{b}}$, Manuel Collet ${ }^{\mathrm{a}}$ \\ a FEMTO-ST Institute, Department of Applied Mechanics, 24, rue de l'épitaphe, 25000 Besançon, France \\ b LTDS, École Centrale de Lyon, 36 avenue Guy de Collongue, 69134 Écully, France
}

\begin{abstract}
Porous materials are used in many vibroacoustic applications. Different available models describe their behaviors according to materials' intrinsic characteristics. For instance, in the case of porous material with rigid frame, and according to the Champoux-Allard model, five parameters are employed. In this paper, an investigation about this model sensitivity to parameters according to frequency is conducted. Sobol and FAST algorithms are used for sensitivity analysis. A strong parametric frequency dependent hierarchy is shown. Sensitivity investigations confirm that resistivity is the most influent parameter when acoustic absorption and surface impedance of porous materials with rigid frame are considered. The analysis is first performed on a wide category of porous materials, and then restricted to a polyurethane foam analysis in order to illustrate the impact of the reduction of the design space. In a second part, a sensitivity analysis is performed using the Biot-Allard model with nine parameters including mechanical effects of the frame and conclusions are drawn through numerical simulations.
\end{abstract}

\section{Introduction}

Porous materials are used in a variety of acoustic applications. Prediction tools of acoustic characteristics for these materials are necessary. Zwikker and Kosten [1]and Biot [2,3] developed the first popular porous media models. A thorough review of these models and further developments was performed by Attenborough [4]. For the case of rigid frame porous media, Allard [5,6] developed a five parameters model, based on the idea of Johnson [7,8]. Measurement and identification of characteristics can be difficult and time consuming, and understanding the model sensitivity can make the optimization of the sound packages easier and facilitate new concept developments. Only very few papers in the open literature on porous media deal with sensitivity of models. Some elements about first-order estimation of impact of parameters are presented for instance in [9]. This gives a useful information, which remains limited to very small variations of parameters without considering any coupling effect between them. To the author's knowledge, one of the most advanced studies on the sensitivity analysis of porous materials models has been proposed by Bolton et al. [10,11], in which a singular value decomposition is performed on the so-called sensitivity matrix, which is build from first-order estimation of derivatives (finite differences) and concatenates effects on absorption coefficient or transmission loss factor for different frequencies. A Singular Value Decomposition is then performed to check the coupling effects between the parameters (the parameters being considered as independent) in order to reduce the size of the design space for identification purpose. The aim of this contribution is to go one step further, by applying rigorous sensitivity analysis

\footnotetext{
* Corresponding author.

E-mail address: morvan.ouisse@univ-fcomte.fr (M. Ouisse).
} 
techniques to porous material models. For illustration purpose, the main features of interest are the acoustic impedance and the absorption coefficient of a sample of porous material backed by an impervious rigid wall. The model used for the description of the acoustic performances is the Champoux-Allard one (depending on five parameters: porosity, flow resistivity, tortuosity, viscous and thermal characteristic lengths). It should be noticed that the methodology is general and can be applied to more complicated porous material models. For instance, the considered sensitivity approach is also applied in this paper using the Biot-Allard poroelastic model.

In this paper we focus on global sensitivity analysis techniques. We classically distinguish two families of methods, namely the local and the global ones. Local sensitivity techniques are low cost, very easy to implement, but they are only able to capture the sensitivity of the model in a limited subset of the design space. On the other hand, global sensitivity analyses, which require a larger computational cost, give information about sensitivity which are valid for the whole design space and can deal with interactions effects between parameters. Sobol and FAST global sensitivity methods are considered here. The main issue is to clarify how the variability associated with the model inputs affects the model outputs [12]. Sensitivity analysis is also expected to (but not limited to) determine which input parameters contribute the most to output variabilities [13]; which parameters are insignificant; and estimate parameter interactions.

A comprehensive review of the different sensitivity analysis methods, including their advantages and drawbacks, has been proposed by Helton et al. [14] and Frey et al. [15]. A comparison of these methods can be found in [16-20]. Among the available sensitivity analysis methods, we propose in this paper to apply Sobol [21] and FAST [22].

The paper is structured as follows. Section 2 provides a brief survey of the sensitivity analysis methods considered in the paper. Section 3 recalls the porous material models used in this work. In Section 4, the Champoux-Allard model is first considered. Sensitivity results are presented and discussed. This analysis is performed considering a large design space whose parameters represent a wide variety of porous materials. Some remarks are drawn about agreements between Sobol and FAST results. Then, a comparison is performed between the first-order sensitivity and total sensitivity indexes, in order to evaluate interaction effects vs. total ones. In Section 5 , another sensitivity analysis is performed when the design space is limited to a specific porous material, namely a polyurethane foam. In particular, a focus is made on the choice of the probability functions used in the sensitivity analysis. Some comments related to modelling and characterization of porous materials are given. Sections 6 and 7 are dedicated to sensitivity analysis of materials that exhibit fluid-structure coupled behavior in the frequency range of interest. To that end, the Biot-Allard model is considered, first with a large porous materials data base, then a restriction to a given type of material. Finally, some recommendations and concluding remarks are given in Section 8.

\section{Sensitivity analysis methods}

Most engineering problems use parametric models. Sensitivity analysis provides tools to understand the impact of each parameter on the outputs of interest. Associated methods are classically divided into two categories, namely the local sensitivity analysis techniques and the global ones. The local methods estimate the sensitivity of a given model to input parameters using different orders of partial derivatives. This type of analysis is limited to small variations of parameters and is not able to capture the coupling effects between them. The most popular technique in that category is the One-At-aTime (OAT) technique, which basically evaluates the sensitivity of a feature $f$ to a parameter $x$ using the partial derivative $\partial f / \partial x$ which is estimated through finite differences $\Delta f / \Delta x$. This kind of technique is widely used and generally gives pertinent information in a local point of view.

Global sensitivity analysis [12] is required when large design space and/or coupling effects are concerned. Among possible methods the "importance measure method" or "correlation ratio technique" are capable of estimating the contribution of each parameter to the output variance [23]. However, whether a parameter is influential or not depends also on the interactions and influences of all the parameters. In this paper, two global methods are considered, namely the Fourier Amplitude Sensitivity Test (FAST) and Sobol methods, which not only can measure the "main effect" (also named first-order term) but can also compute the so-called "Total Sensitivity Indexes" (TSI) [12]. The brief outline of global sensitivity estimation is largely inspired from different references and cookbooks offered by Saltelli and Tarantola [24].

\subsection{Sobol-variance analysis}

Indeed, concerning a quantity of interest (the output of the model), the Total Sensitivity Index of parameter $i$, denoted by $\operatorname{TSI}(i)$, is defined as the sum of all the sensitivity indexes (including all the interactions effects) involving parameter $i$. For example, suppose that we only have three input parameters $(A, B$ and $C$ ) in our model. The total effect of parameter $A$ on the output is $\operatorname{TSI}(A)=\operatorname{SI}(A)+\operatorname{SI}(A B)+\operatorname{SI}(A C)+\operatorname{SI}(A B C)$. Here, $\operatorname{SI}(A)$ denotes the first-order sensitivity index for parameter $A, \operatorname{SI}(A X)$ is the second-order sensitivity index for the parameters $A$ and $X$ (for $X \neq A$ ), i.e. the interaction between parameters $A$ and $X$, and so on. The first-order sensitivity index does not take into account coupling effects between parameters, but considers variation of the parameter according to its statistical distribution on a possibly large range, in that sense it is more general than the classical OAT finite differences based index.

In general, a mathematical model denoted $f(\cdot)$ is a plant connecting a set of $n$ input parameters to an output $y$, namely $y=f(x)$, where $x$ is a random vector of input parameters, characterized by a joint probability density function 
$p(x)=p\left(x_{1}, x_{2}, \ldots, x_{n}\right)$. Statistics of the output, $y$, can be computed from the $r$ th moment, which is given by

$$
E\left(y^{r}\right)=\int_{K^{n}} f^{r}\left(x_{1}, x_{2}, \ldots, x_{n}\right) p\left(x_{1}, x_{2}, \ldots, x_{n}\right) \mathrm{d} x,
$$

where $K^{n}$ is the $n$-dimensional space of the input parameters. The computation of integral in (1) gives sensitivity measures of different types. In the Sobol approach, the idea for the sensitivity indexes estimation is the decomposition of the function $f(x)$ into summands of increasing dimensionality [21], namely

$$
f\left(x_{1}, \ldots, x_{n}\right)=f_{0}+\sum_{i=1}^{n} f_{i}\left(x_{i}\right)+\sum_{i=1}^{n} \sum_{j=i+1}^{n} f_{i, j}\left(x_{i}, x_{j}\right)+\cdots+f_{1, \ldots, n}\left(x_{1}, \ldots, x_{n}\right) .
$$

Sobol [21] showed that the decomposition (2) is unique and that all the terms in (2) can be evaluated via multidimensional integrals, namely $f_{0}=\int_{K^{n}} f(x) \mathrm{d} x, f_{i}\left(x_{i}\right)=-f_{0}+\int_{K^{n \sim i}} f(x) \mathrm{d} x_{\sim i}$ and $f_{i j}\left(x_{i}, x_{j}\right)=-f_{0}-f_{i}\left(x_{i}\right)-f_{j}\left(x_{j}\right)+\int_{K^{n} \sim\{i, j\}} f(x)$ $\mathrm{d} x_{\sim\{i, j\}}$ with the convention that $\int_{K^{n \sim i}} \mathrm{~d} x_{\sim i}$ and $\int_{K^{n} \sim\{i, j\}} \mathrm{d} x_{\sim\{i, j\}}$ denote the integration over all parameters except $x_{i}$, and $x_{i}$ and $x_{j}$, respectively. Here, “ $\sim$ " means "complementary of". Analogous formulas can be obtained for the higher-order terms. The variance based sensitivity indexes follow naturally this concept; the total variance $D$ of $f(x)$ is then defined to be

$$
D=\int_{K^{n}} f^{2}(x) \mathrm{d} x-f_{0}^{2},
$$

while partial variances are computed from each of the terms in (2) namely

$$
D_{i_{1}, \ldots, i_{s}}=\int_{0}^{1} \ldots \int_{0}^{1} f_{i_{1}, \ldots, i_{s}}^{2}\left(x_{1}, \ldots, x_{s}\right) \mathrm{d} x_{1} \ldots \mathrm{d} x_{s} .
$$

In the Sobol approach, the integrals in (3) and in (4) can be evaluated with a Monte Carlo (MC) integral.

The sensitivity indexes can then be evaluated by

$$
\operatorname{SI}\left(x_{1} \ldots x_{s}\right)=\frac{D_{i_{1}, \ldots, i_{s}}}{D}
$$

and the total sensitivity index of parameter $x_{i}$ on output $y$ is the sum of all sensitivity indexes $\operatorname{SI}(\kappa)$ where $\kappa$ involves $x_{i}$.

\subsection{The FAST technique}

The Fourier Amplitude Sensitivity Test (FAST) [22,25,26] has first been developed for chemical applications. It is an elegant way to estimate properly the first-order effects (i.e. without parameters interactions) and with a low calculation cost. It is based on the idea that the previous multidimensional integrals can be converted in one-dimensional ones using a transformation such as

$$
x_{i}=\frac{1}{2}+\frac{1}{\pi} \arcsin \left(\sin \left(\omega_{i} s+\varphi_{i}\right)\right)
$$

for each parameter $i$, where $s \in(-\pi, \pi)$ is a scalar variable whose evolution is driven by the probability density function of the variable, $\omega_{i}$ is a properly chosen angular frequency (see [25] for details) in order to avoid interferences between spectral components of parameters, and $\varphi_{i}$ is a random phase-shift which allows different realizations of the parameter space. This resampling strategy was not considered in the initial FAST technique, it has been included in an improved version some years later [17].

The total variance of $f$ is then approximated by

$$
D \approx 2 \sum_{j=1}^{\left(N_{s}-1\right) / 2}\left(A_{j}^{2}+B_{j}^{2}\right)
$$

where $N_{s}$ is the number of samples, while $A_{j}$ and $B_{j}$ are the Fourier coefficients defined as

$$
\begin{aligned}
A_{j} & =\frac{1}{2 \pi} \int_{-\pi}^{\pi} f\left(x_{1}, x_{2}, \ldots, x_{n}\right) \cos (\mathrm{j} s) \mathrm{d} s, \\
B_{j} & =\frac{1}{2 \pi} \int_{-\pi}^{\pi} f\left(x_{1}, x_{2}, \ldots, x_{n}\right) \sin (\mathrm{j} s) \mathrm{d} s .
\end{aligned}
$$

The first-order effect associated to the parameter $i$ is obtained by

$$
D_{i} \approx 2 \sum_{j=1}^{M}\left(A_{\mathrm{j} \omega_{i}}^{2}+B_{\mathrm{j} \omega_{i}}^{2}\right),
$$

where $M$ is the interference order corresponding to the chosen set of frequencies $\omega_{i}$.

The initial version was only able to capture the first-order global effects (without interactions), at a very low calculation cost, since only one set of sample was required. This technique has been improved later [17] to include estimation of total sensitivity indexes, but requires a set of samples for each parameter. The total effect of a parameter on variance is then 
captured using another set of frequencies, by computing difference between unity and the variance estimated when all parameters vary but the one of interest.

\section{Porous materials models}

In this section, porous material models are briefly recalled. For further details and developments, readers are advised to check published textbooks, in particular the one by Allard and Atalla [5]. In this paper, the analysis is restricted to acoustic properties of porous materials as outputs for the sensitivity analysis. More precisely, the acoustic impedance and corresponding absorption coefficient at normal incidence are considered. These quantities are estimated for a sample of given thickness $e$ backed by an impervious rigid wall. The lateral dimensions are taken infinite. Both rigid and elastic frames are considered in what follows for sensitivity purposes.

\subsection{Johnson-Allard, simplified Lafarge and Champoux-Allard models}

The first model considered in this paper corresponds to cases in which the frame of the porous material remains rigid. In this context, wave propagation is characterized by the effective density $\rho_{e}$ and bulk modulus $K$ of the material or using the characteristic impedance $Z_{c}$ and wavenumber $k$ such that

$$
\begin{gathered}
k(\omega)=\omega \sqrt{\frac{\rho_{e}(\omega)}{K(\omega)}}, \\
Z_{c}(\omega)=\sqrt{\rho_{e}(\omega) K(\omega)},
\end{gathered}
$$

$\omega$ being the angular frequency. The wavenumber and the characteristic impedance can be used to evaluate the surface impedance $\left(Z_{s}\right)$ and the absorption coefficient $(\alpha)$ when the sample is backed by an impervious rigid wall:

$$
\begin{gathered}
Z_{s}=-\frac{1}{\phi} \mathrm{j} Z_{c} \cot (k e), \\
\alpha=1-\left|\frac{Z_{s}-Z_{0}}{Z_{s}-Z_{0}}\right|^{2},
\end{gathered}
$$

where $\phi$ is the porosity, $\mathrm{j}=\sqrt{-1}, e$ is the thickness of the sample, $Z_{0}=\rho_{0} c_{0}$ is the specific impedance of the fluid saturating the pores (typically air, $\rho_{0}$ being its density and $c_{0}$ the speed of sound).

The model used in this paper is the Champoux-Allard model, which is detailed in Appendix A. It requires the knowledge of five material parameters, namely the porosity $\phi$, the flow resistivity $\sigma$, the tortuosity $\alpha_{\infty}$, the characteristic viscous and thermal lengths $\Lambda, \Lambda^{\prime}$.

\subsection{Biot-Allard model}

For porous materials exhibiting mechanical resonances of the frame in the frequency range of interest, the generalized Biot model (also called Biot-Allard model) has to be considered. In this model, nine parameters are associated to the fluidstructure behavior of the porous media: the porosity $\phi$, the flow resistivity $\sigma$, the tortuosity $\alpha_{\infty}$, the characteristic viscous and thermal lengths $\Lambda, \Lambda^{\prime}$ for the fluid phase, and Young's modulus $E$, density $\rho_{S}$, Poisson coefficient $v$, loss factor $\eta$ for the solid phase (considered to be isotropic). In order to improve readability of this paper, the numerous equations of this model are not recalled here. They can be found in several papers and textbooks, in particular in Ref. [5].

\section{Detailed sensitivity analysis of Champoux-Allard model}

In this paper, the models $f(\cdot)$ considered for sensitivity analysis are, respectively, the real and imaginary parts of surface impedance and the acoustic absorption coefficient. Input parameters are the five parameters according to the ChampouxAllard model: porosity, flow resistivity, tortuosity, viscous and thermal characteristics lengths $\left(\phi, \sigma, \alpha_{\infty}, \Lambda, \Lambda^{\prime}\right)$. Table 1 gives lower and upper bounds of each parameter. The probability density functions used in this first analysis are uniform, the impact of this choice will be discussed later in the paper.

Lower and upper limits in Table 1 were determined according to collected industrial data and published works, representing a wide variety of porous materials. The objective is to characterize the sensitivity of the model while covering a large part of the porous materials used in practical applications. It is well known that, for a given porous material, all the parameters are not independent: they mainly depend on the microstructure. Several techniques can be used to link its geometry to the parameters considered here. Nevertheless, in this first analysis, the aim is to check the impact of each parameter separately, independently from the microstructure geometry. Clearly the link between the parameters is changing with the geometry of the microstructure, and considering all possible materials together in this first step can be somehow interpreted as a decoupling between the parameters. Among all samples that will be used in the simulations due 
Table 1

Lower and upper bounds of input parameters.

\begin{tabular}{llll}
\hline Parameter & Variable & Lower bound & Upper bound \\
\hline Porosity & $\phi(-)$ & 0.7 & 0.99 \\
Flow resistivity & $\sigma\left(\mathrm{N} \mathrm{s} \mathrm{m}^{-4}\right)$ & 1500 & 200000 \\
Tortuosity & $\alpha_{\infty}(-)$ & 1 & 3 \\
Viscous characteristic length & $\Lambda(\mu \mathrm{m})$ & 5 & 200 \\
Thermal characteristic length & $\Lambda^{\prime}(\mu \mathrm{m})$ & 5 & 400 \\
\hline
\end{tabular}

to random sampling in the bounds of interest, a part of them do not correspond to any existing material. This is not an issue, since the question that we want to answer here is not related to a specific material, but the reader has to keep this point in mind. Another way to understand the philosophy of this analysis is to consider a blind identification of the foam parameters, without knowledge of the nature of the material: in this case the parameters can be considered as independent from the user's point of view, and the performed analysis gives information about the possibility to identify a given parameter among all ones. In the next section of this paper we will focus on a specific material.

A similar study could be conducted using microstructure related parameters, but it would certainly be much more restrictive in the sense that only a finite number of geometric configurations could be considered. Nevertheless, for the foam of interest, it would give very pertinent information about impact of the considered parameters.

The sensitivity analysis has been conducted using both Sobol and FAST methods. This investigation has been conducted in the frequency band [ $100 \mathrm{~Hz}-2500 \mathrm{~Hz}$ ] with $25 \mathrm{~Hz}$ frequency sampling. The input parameters sensitivity indexes are then estimated at each frequency step. A $25 \mathrm{~mm}$ thick porous material sample was considered (influence of the thickness value will be discussed in Section 7). Sensitivity methods efficiency is closely related to the number of samples and number of parameters, but also the number of repetitions for Sobol. It is important to remind that Sobol method gives all partial sensitivity indexes, while the FAST technique gives only total and first sensitivity indexes (STI and SI) for each input parameter. The number of samples must be as low as possible to keep a low computational cost, but large enough to ensure the convergence of the analysis. These parameters are closely related to the number of parameters, to their statistical properties, and to the complexity of the outputs of interest.

In the considered case, FAST method needs 100000 data points for convergence. Sobol needs 10 repetitions of 70000 computation data points. In this case, data points are chosen using Monte Carlo method.

\subsection{Sobol vs. FAST}

In this subsection, sensitivity indexes of parameters on acoustic performances are given for some chosen frequencies (namely $500 \mathrm{~Hz}$ and $2000 \mathrm{~Hz}$ ). Comparison is done between FAST and Sobol methods, respectively, between first and total orders sensitivity indexes. Table 2 shows the results. For instance, in the case of real part of impedance at $2000 \mathrm{~Hz}$, the computation with FAST method allows quantification of first-order sensitivity indexes for the five parameters. Flow resistivity is the most influencing and its index is about $46 \%$, then viscous characteristic length index with $42 \%$, then tortuosity, thermal characteristic length, and porosity with very low indexes which indicates that their first-order effect is almost negligible compared to $\sigma$ and $\Lambda$ considering the bounds of interest. Sensitivity indexes in Table 2 show that FAST and Sobol methods are in good agreement, with small numerical discrepancies appearing for some low values or for the total sensitivity index. Nevertheless, the ranking of parameters influence is identical. The information given by the total sensitivity index will be further investigated in the next section. For the case of first-order sensitivity index, Sobol and FAST indexes are equivalent to the so-named Standardized Regression Rank Coefficients (SRRC) defined in [23]. Obviously, FAST method takes less time than Sobol which needs repeating computation $p$-times. Sensitivity indexes obtained by FAST method are used thereafter, considering that the first and total indexes give pertinent information for understanding the impact of each parameter separately and to check the effects of the couplings with other parameters.

\subsection{SI vs. TSI}

In this section, the analysis concentrates on the comparison between first-order and total sensitivity indexes, of course according to frequency, obtained with the FAST technique. Fig. 1 shows sensitivity index evolutions for each parameter (five columns concerning, respectively, porosity, flow resistivity, tortuosity, viscous and thermal characteristic lengths), and for each output of interest (three rows concerning, respectively, real and imaginary parts of the surface impedance, and the acoustic absorption coefficient).

Fig. 1 shows that total sensitivity of flow resistivity on the real part of surface impedance globally decreases from $90 \%$ to $40 \%$ when the frequency increases from $100 \mathrm{~Hz}$ to $2500 \mathrm{~Hz}$. This tendency is inverted at very low frequency. The total sensitivities of porosity, tortuosity, and viscous characteristic lengths on the real part of surface impedance, increase, roughly. Up to $2000 \mathrm{~Hz}$, flow resistivity is the most important parameter, and above that frequency, viscous characteristic length takes the pole position. Thus, the strong dependency of sensitivity indexes according to frequency does not allow a simple hierarchy of parameters. Concerning the imaginary part of the surface impedance, the porosity is preponderant in 
Table 2

Sobol and FAST sensitivity indexes of parameters on acoustic performances (Champoux-Allard model).

\begin{tabular}{|c|c|c|c|c|c|c|c|c|}
\hline Output & Sensitivity index & Frequency $(\mathrm{Hz})$ & Sensitivity method & $\phi$ & $\sigma$ & $\alpha_{\infty}$ & $\Lambda$ & $\Lambda^{\prime}$ \\
\hline \multirow[t]{8}{*}{$\operatorname{Re}\left(Z_{S}\right)$} & \multirow[t]{4}{*}{ First-order sensitivity index } & \multirow[t]{2}{*}{500} & FAST & 0.004 & 0.926 & 0.003 & 0.039 & 0.028 \\
\hline & & & Sobol & 0.006 & 0.847 & 0.005 & 0.086 & 0.028 \\
\hline & & \multirow[t]{2}{*}{2000} & FAST & 0.031 & 0.465 & 0.079 & 0.418 & 0.007 \\
\hline & & & Sobol & 0.024 & 0.364 & 0.063 & 0.389 & 0.004 \\
\hline & \multirow[t]{4}{*}{ Total sensitivity index } & \multirow[t]{2}{*}{500} & FAST & 0.061 & 0.912 & 0.069 & 0.122 & 0.083 \\
\hline & & & Sobol & 0.006 & 0.859 & 0.026 & 0.121 & 0.026 \\
\hline & & \multirow[t]{2}{*}{2000} & FAST & 0.085 & 0.515 & 0.147 & 0.527 & 0.057 \\
\hline & & & Sobol & 0.039 & 0.472 & 0.115 & 0.529 & 0.015 \\
\hline \multirow[t]{8}{*}{$\operatorname{Im}\left(Z_{S}\right)$} & \multirow[t]{4}{*}{ First-order sensitivity index } & \multirow[t]{2}{*}{500} & FAST & 0.317 & 0.197 & 0.030 & 0.180 & 0.275 \\
\hline & & & Sobol & 0.275 & 0.167 & 0.022 & 0.227 & 0.237 \\
\hline & & \multirow[t]{2}{*}{2000} & FAST & 0.001 & 0.925 & 0.044 & 0.021 & 0.009 \\
\hline & & & Sobol & 0.002 & 0.809 & 0.041 & 0.046 & 0.006 \\
\hline & \multirow[t]{4}{*}{ Total sensitivity index } & \multirow[t]{2}{*}{500} & FAST & 0.358 & 0.266 & 0.116 & 0.281 & 0.318 \\
\hline & & & Sobol & 0.288 & 0.190 & 0.056 & 0.281 & 0.245 \\
\hline & & \multirow[t]{2}{*}{2000} & FAST & 0.041 & 0.895 & 0.128 & 0.135 & 0.050 \\
\hline & & & Sobol & 0.006 & 0.873 & 0.100 & 0.135 & 0.013 \\
\hline \multirow[t]{8}{*}{$\alpha$} & \multirow[t]{4}{*}{ First-order sensitivity index } & \multirow[t]{2}{*}{500} & FAST & 0.133 & 0.539 & 0.029 & 0.232 & 0.066 \\
\hline & & & Sobol & 0.105 & 0.428 & 0.025 & 0.232 & 0.057 \\
\hline & & \multirow[t]{2}{*}{2000} & FAST & 0.011 & 0.827 & 0.007 & 0.148 & 0.007 \\
\hline & & & Sobol & 0.010 & 0.666 & 0.007 & 0.142 & 0.008 \\
\hline & \multirow[t]{4}{*}{ Total sensitivity index } & \multirow[t]{2}{*}{500} & FAST & 0.165 & 0.617 & 0.098 & 0.372 & 0.132 \\
\hline & & & Sobol & 0.104 & 0.572 & 0.058 & 0.377 & 0.077 \\
\hline & & \multirow[t]{2}{*}{2000} & FAST & 0.044 & 0.803 & 0.136 & 0.310 & 0.041 \\
\hline & & & Sobol & 0.014 & 0.786 & 0.109 & 0.301 & 0.014 \\
\hline
\end{tabular}

the low frequency range, while flow resistivity is the most influent parameter in the whole frequency range. Other parameters have relatively small indexes. Total effect of flow resistivity on acoustic absorption coefficient varies between $60 \%$ and $80 \%$ along frequency scale, while sensitivity indexes of viscous characteristic length and tortuosity vary between $20 \%$ and 50\%, and other parameters have lower indexes. Sensitivity results provided here are in concordance with the empirical Delany and Bazley model [27] in which the characteristic impedance and wavenumber depend only on flow resistivity and frequency. For example, the porosity is found to have a very low impact on the acoustic impedance. This kind of result leads to comments:

- The value of this parameter has a limited impact on the estimated absorption coefficient: approximate value can be accepted with confidence. The physical reason is that the feature of interest (absorption coefficient for normal incident waves) is a global indicator. The results would have certainly been different if the features would have been related to the local behavior of the material: in our case, very precise description of the local effects is not required to obtain confident information on the global behavior of interest.

- In terms of identification procedures, this result implies that testing the sample measuring only absorption coefficient related to plane normal incident waves will not allow a confident identification of this parameter. In the considered case, it is expected that the flow resistivity and the viscous characteristic length can be easily identified, while since other parameters' sensitivities are very small, they will require a special attention for efficient identification: the tortuosity will be provided by a detailed analysis around $1000 \mathrm{~Hz}$, while the thermal length will be efficiently identified only in the very low frequency range. As said above, the precision obtained on the porosity is expected to be poor since its indexes are almost always lower than $10 \%$.

As already mentioned, these results are obtained considering a wide variety of porous materials, and for a fixed thickness of the sample. The conclusions are then restricted to this context. In particular, remarks established concerning the identification aspects are valid if the analyst has no idea about the values of the parameters of the sample. In the next section, we will illustrate the sensitivity analysis using a reduced design space, i.e. the case in which the possible variations on parameters' values are restricted to limited ranges.

Analyzing SIs vs. TSIs (Fig. 1), it is clear that Total Sensitivity Indexes (TSIs) are superior to first-order Sensitivity Indexes (SIs) regardless frequency, because of coupling effects between parameters. This is due to the fact that total index of a parameter is the sum of first-order index and interactions (involving this parameter), and allows to appreciate over-than order two sensitivity indexes compared with first-order effects. Nevertheless, as indicated in [17], this is only one of the ways to define the total sensitivity of a parameter. In the case of real and imaginary parts of the surface impedance and acoustic absorption coefficient, interactions between flow resistivity and the other parameters can reach, respectively, $20 \%$, 

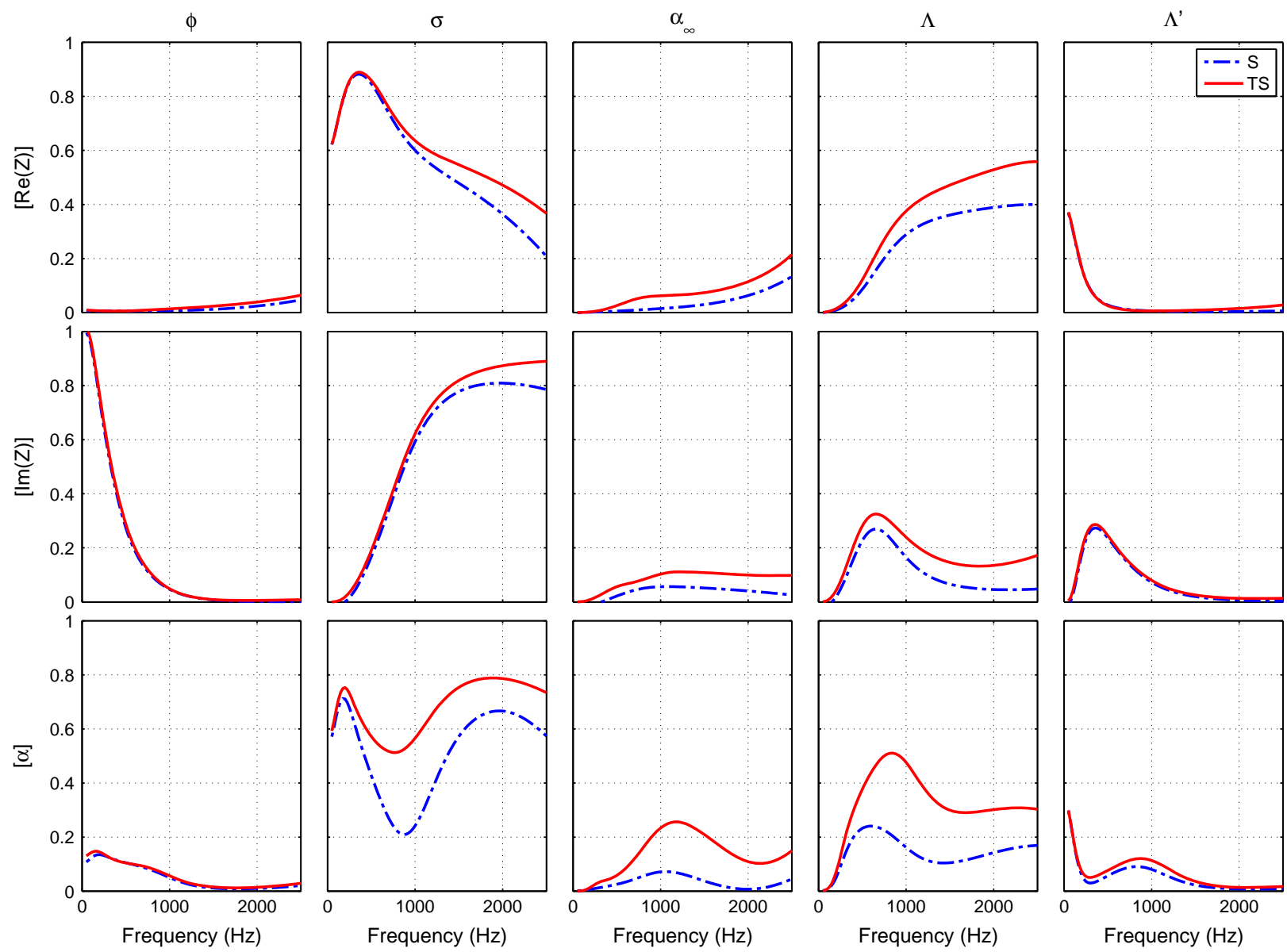

Fig. 1. First-order (SI) and total sensitivity (TSI) indexes of porous material parameters: effects on surface impedance and acoustic absorption.
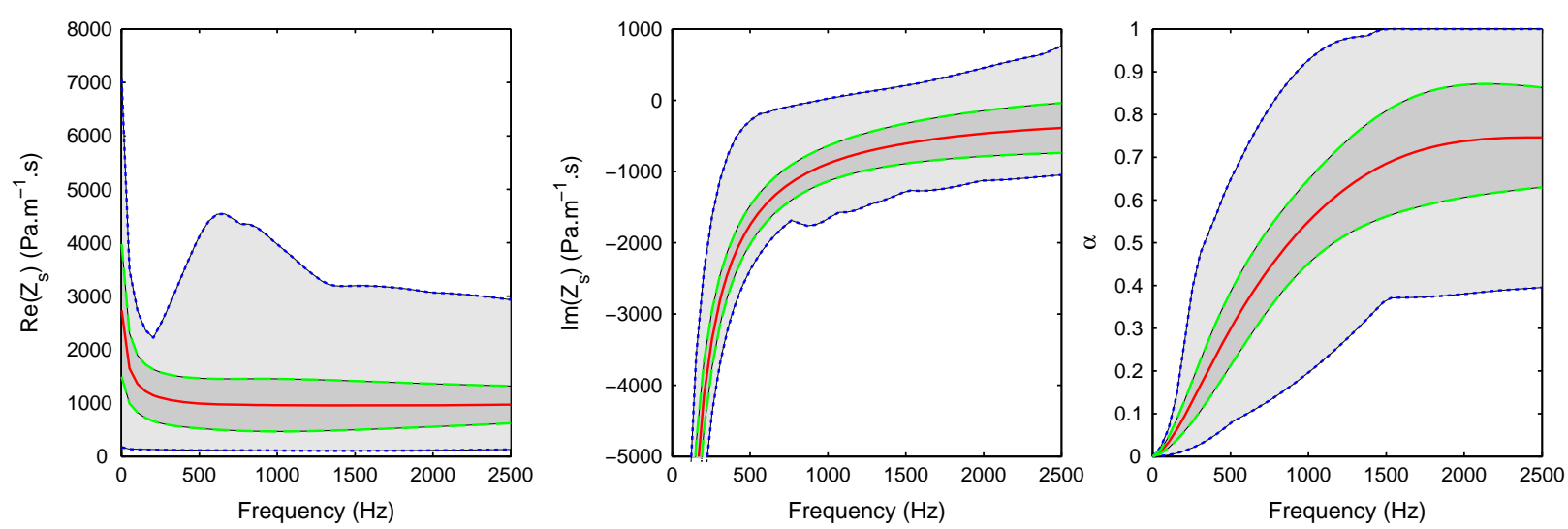

Fig. 2. Statistics of the set of outputs: mean value, mean value \pm standard deviation, minimum and maximum values.

$13 \%$ and $40 \%$ of $\operatorname{TSI}(\phi)$. Other strong interactions can appear with tortuosity and viscous characteristic length depending on the frequency range of interest.

The previous remarks are based on the sensitivity indexes defined in part 2. It is important to bear in mind that these indexes are related to a unit total sensitivity, and the conclusion must be weighted by the absolute value of the sensitivity: a parameter could be detected as the main source of sensitivity (i.e. with a TSI close to 1), but still induce small changes on the feature of interest. The absolute value of total sensitivity is given by the analysis. In the considered case, since the input parameters ranges are very large, the impact on features is also very important. This fact is illustrated in Fig. 2, which 
shows the mean value of features, together with minimum, maximum values, and also the mean value plus or minus the standard deviation which can be considered as a global measure of total sensitivity.

It is then clear that the conclusion presented above must be weighted. We propose here to do this using the normalized standard deviation for outputs of interest, namely the ratio of the standard deviation to the mean value:

$$
\mathrm{NSD}(y)=\frac{\sqrt{E\left(y^{2}\right)-E(y)^{2}}}{E(y)} .
$$

In the considered case, this corresponds to the three curves given in Fig. 3, which indicates that the global sensitivity of real part of impedance is almost constant in the frequency range of interest, while for imaginary part it is low at low frequency and quickly grows with frequency (up to 0.9 , which corresponds to a very high sensitivity). For absorption coefficient, the normalized standard deviation is high in the low frequency range and decreases with frequency.

\subsection{Parameters classification}

Figs. 1 and 3 can be represented in a single one to improve readability, as illustrated in Fig. 4 .

This compact representation allows the refinement of the conclusions which were made above by only considering the sensitivity indexes: for example, for identification purpose, it was observed that, in the low frequency range, the porosity was driving almost all the sensitivity of the imaginary part of the impedance. As said before, this indicates that it is in this frequency range that the identification of the porosity would be the most confident. Nevertheless, when combining this with the normalized standard deviation which indicates that, globally, the value of this indicator is very low at low frequency, this indicates that even if all the sensitivity is due to the porosity, the identification will not be easy because of the 0.1 value of NSD. Both information are then important and must be considered for identification purpose and global sensitivity conclusions.

A qualitative analysis of the impact of each parameter on the outputs of interest can then be performed. These conclusions are given according to the hypotheses made before, in particular concerning the ranges considered for each parameter, the outputs of interest and the fixed thickness of the material:

- the porosity has a very limited impact on the features of interest. An exception occurs at very low frequency (under $800 \mathrm{~Hz}$ ) for the imaginary part of the impedance, where it drives almost all the sensitivity, even if in this frequency range, the standard deviation of the output remains qualitatively low;

- the flow resistivity is globally the most sensitive parameter, for each feature and on the whole frequency range;

- the tortuosity has a very limited impact on acoustic performances, except around $1000 \mathrm{~Hz}$ where absorption coefficient is quite sensitive on coupling effects between tortuosity, thermal characteristic length and flow resistivity;

- the viscous characteristic length is almost irrelevant under $500 \mathrm{~Hz}$, and then becomes much more important above that value: it is globally the second most important parameter in terms of sensitivity, and even the first one after $2000 \mathrm{~Hz}$ for real part of surface impedance;

- the thermal characteristic length exhibits quite large sensitive effects below $200 \mathrm{~Hz}$ on real part of acoustic impedance and absorption coefficient, between $200 \mathrm{~Hz}$ and $500 \mathrm{~Hz}$ for imaginary part of impedance, and around $1000 \mathrm{~Hz}$ on absorption coefficient. For other frequencies, its impact on acoustic performances is very low.

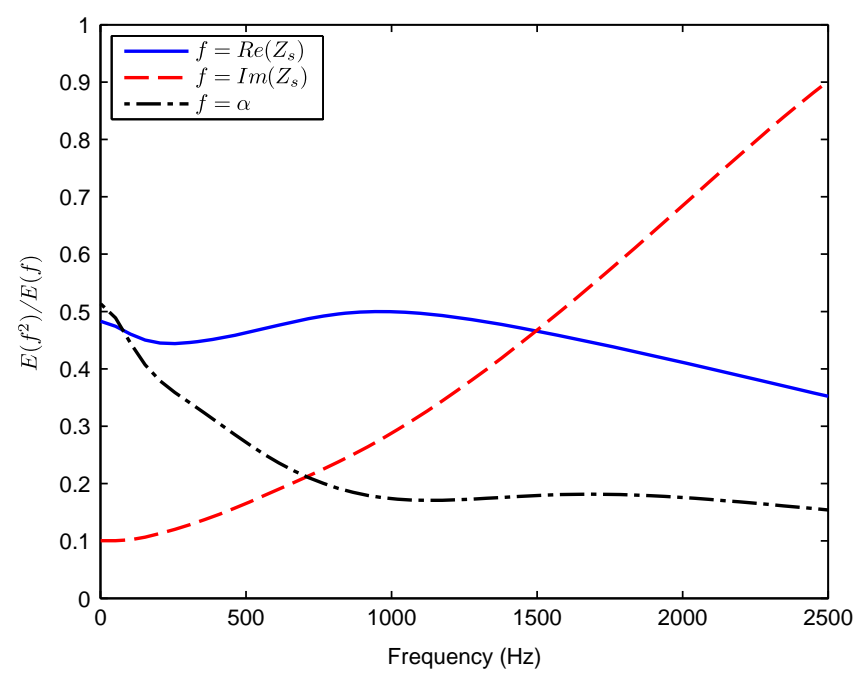

Fig. 3. Normalized standard deviation for outputs of interest. 

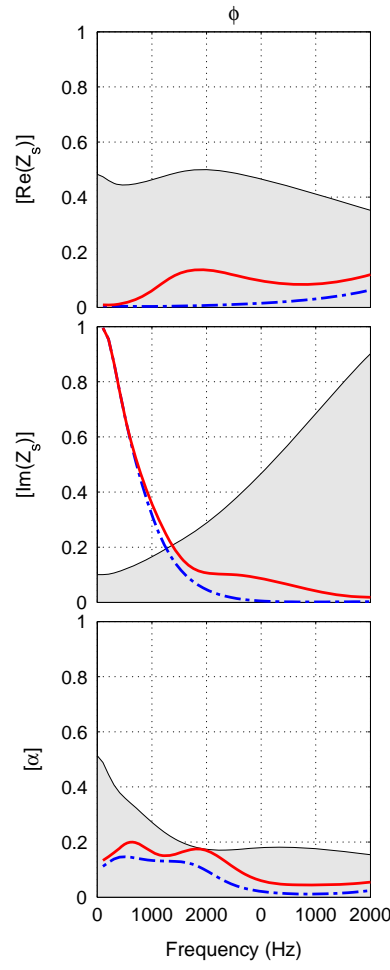
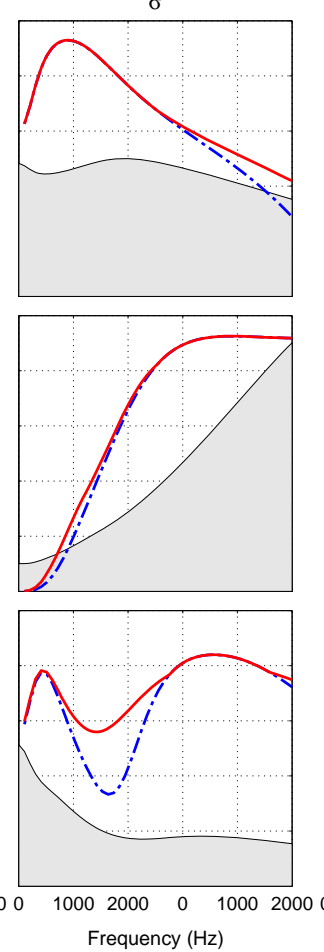
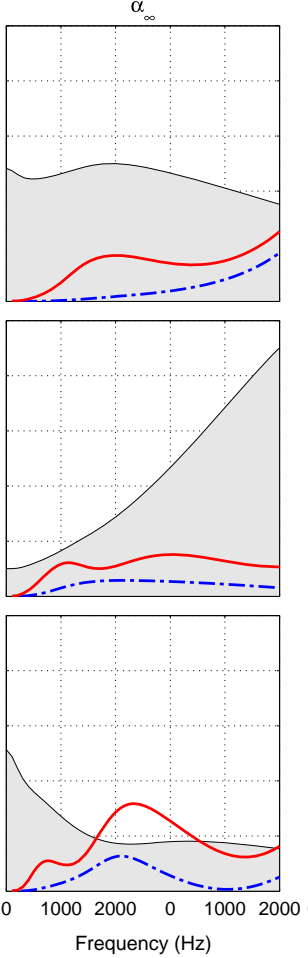

$\Lambda$
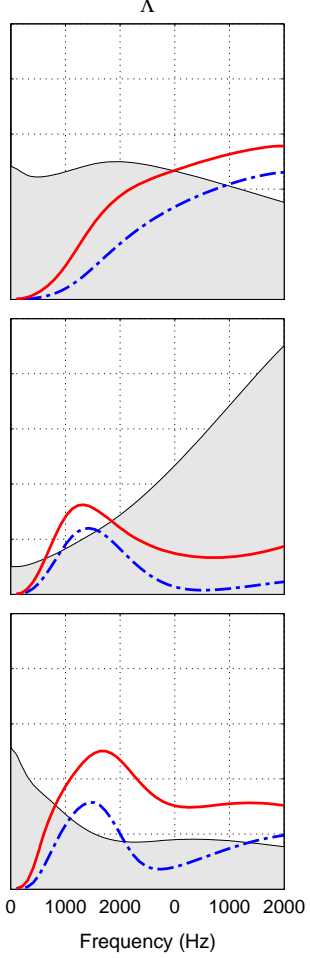
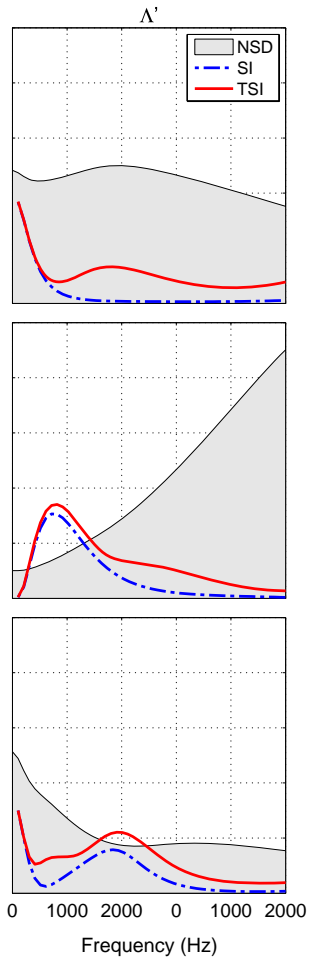

Fig. 4. Normalized standard deviation (NSD), first-order (SI) and total sensitivity (TSI) indexes of porous material parameters: effects on surface impedance and acoustic absorption.

Table 3

Identified values of the parameters of the polyurethane foam [28].

\begin{tabular}{llll}
\hline Parameter & Variable & Lab. 1 & Lab. 2 \\
\hline Porosity & $\phi(-)$ & 0.95 & 0.975 \\
Flow resistivity & $\sigma\left(\mathrm{N} \mathrm{s} \mathrm{m}^{-4}\right)$ & 14750 & 13904 \\
Tortuosity & $\alpha_{\infty}(-)$ & 2.3 & 1.648 \\
Viscous characteristic length & $\Lambda\left(10^{-6} \mathrm{~m}\right)$ & 155 & 77 \\
Thermal characteristic length & $\Lambda^{\prime}\left(10^{-6} \mathrm{~m}\right)$ & 232 & 2.01 \\
\hline
\end{tabular}

\section{Sensitivity analysis of Champoux-Allard model on a specific polyurethane foam sample}

The objective of this section is to show how the previous results are affected when the analysis is restricted to a given material type. This corresponds to a reduction of the size of the design space, in accordance with the knowledge of the considered material. Here a polyurethane foam which has been used and described in Ref. [28] is considered. In this paper, some material parameters identification coming from three different labs are given. A Champoux-Allard model is used, and corresponding data are reproduced in Table 3.

Classically, a basic finite difference analysis can be achieved to have a rough idea about the sensitivity of the model to the parameters' values. Results related to this preliminary investigation on absorption coefficient are presented in Fig. 5. It can be observed that the ordered most sensitive parameters seem to be the viscous characteristic length, then the tortuosity, and the porosity. Other parameter changes slightly affect the value of absorbing coefficient. This type of local approach for sensitivity analysis is limited and is clearly unable to take into account neither coupling effects between parameters nor nonlinear effects.

The global sensitivity analysis is then performed using the improved FAST technique. The variability of the parameters is used to build the probability density functions (pdfs) used in the sensitivity analysis. The lack of information and databases make it difficult to built probability functions with confidence, since at most 3 informations are available for each parameter. Several solutions can then be used to estimate the pdf. Gaussian pdfs should clearly be avoided (parameter's values can be negative, with no physical meaning). Fig. 6 shows several possible pdfs for flow resistivity, identified from the database taken from the literature [28]. This figure shows:

- a basic uniform pdf, which has the advantage to ensure positive values for each parameter, but it does not allow parameters to go outside the region defined by the available data; 
$\phi$

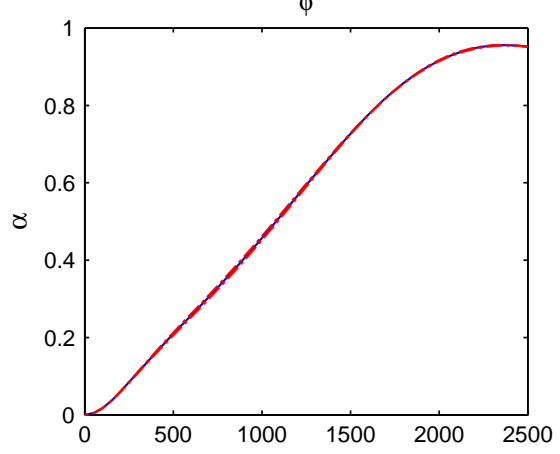

$\sigma$

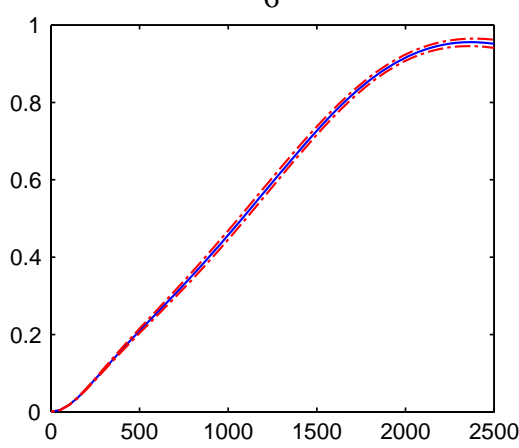

$\alpha_{\infty}$

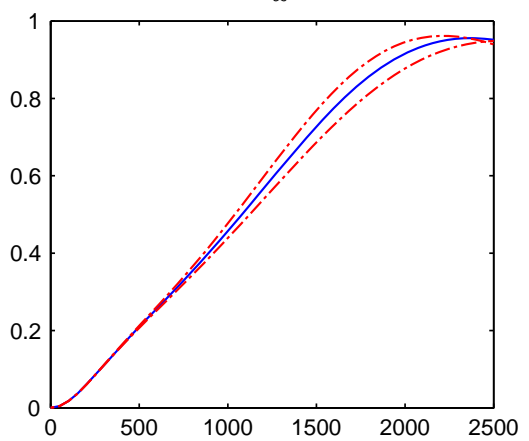

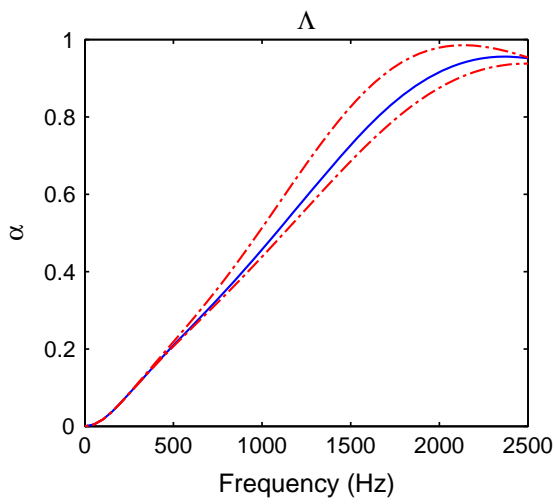

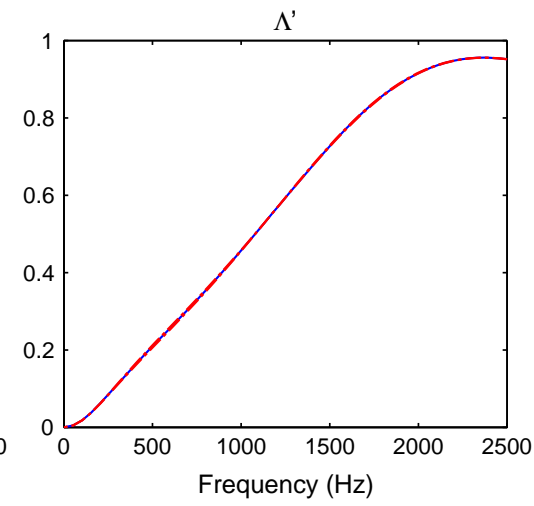

Fig. 5. Basic finite difference sensitivity: absorption coefficient for nominal, minimum and maximum values of each parameter.

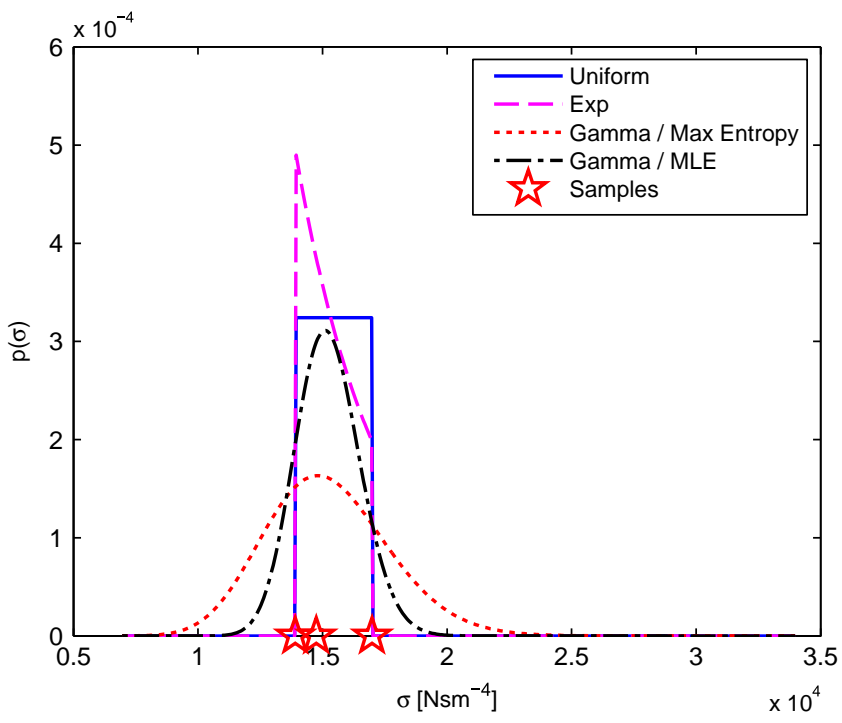

Fig. 6. Examples of pdfs for flow resistivity.

- an exponential pdf, obtained using the principle of maximum entropy [29,30], assuming the samples are confident enough to define bounds and mean of the random variable;

- a gamma distribution, also identified using the principle of maximum entropy, which is more adapted since its bounds are not explicitly given by available samples (the identification is derived by the mean of the samples and the mean of the log of the samples);

- another gamma distribution whose shape and scale parameters can be estimated using a maximum likelihood technique [31] associated to the given data (only the statistical moments, without considering entropy). 
It can be observed that each parameter identification leads to quite different pdfs, and among the chosen shapes, the Gamma distribution is probably the most physical one. Since only few samples are available, there is a large uncertainty in the pdf identification, depending on the knowledge the user has concerning the parameter's uncertainties, and the confidence about given samples.

In particular, one should take into account physical limits of parameters when trying to identify the pdfs: for example, the porosity should obviously be positive and lower than 1, or the tortuosity should not be lower than 1 . In this context, the physical limits must clearly be considered through the use of compact supports for the pdfs. Being given a compact support $[a, b]$, the mean $m_{\phi}$ and the statistical moment of order $2 m_{2}$ (or the standard deviation), the best estimate of the pdf in the sense of the principle of maximum entropy is $[32,30]$

$$
p_{\phi}(x)=\mathbb{1}_{[a, b]}(x) \mathrm{e}^{-\lambda_{0}-x \lambda_{1}-x^{2} \lambda_{2}},
$$

where $\lambda_{0}, \lambda_{1}, \lambda_{2}$ are obtained by minimization of the convex function $\left(\lambda_{0}, \lambda_{1}, \lambda_{2}\right) \mapsto H\left(\lambda_{0}, \lambda_{1}, \lambda_{2}\right)$ where

$$
H\left(\lambda_{0}, \lambda_{1}, \lambda_{2}\right)=\lambda_{0}+\lambda_{1} m_{\phi}+\lambda_{2} m_{2}+\int_{a}^{b} \mathrm{e}^{-\lambda_{0}-x \lambda_{1}-x^{2} \lambda_{2}} \mathrm{~d} x_{1} \mathrm{~d} x_{2}
$$

For the porosity, one obtains a pdf which is quite close to the gamma function, but which ensures that any sample value is lower than 1 (Fig. 7).

The results of both analyzes considering uniform and non-uniform probability density functions are given in Figs. 8 and 9 .

The use of uniform pdfs gives an interesting result: in this case, SIs are almost equal to TSIs. This indicates that the coupling effects between parameters are very low, due to the small parameters possible changes. The ranking which was deduced from the local sensitivity analysis is confirmed for the same reason. Depending on the frequency range of interest, the most sensitive parameters are the flow resistivity and viscous characteristic length. The main difference with the previous analysis concerns tortuosity, which has a strong impact on imaginary part of impedance. One can also point out the impact of this parameter on absorption coefficient around $2500 \mathrm{~Hz}$ : the local sensitivity analysis seems to indicate a very low impact of the parameter, which is confirmed by the corresponding SI index, while the TSI is around $20 \%$, which indicates that large coupling effects occur. Nevertheless, if uniform pdfs are considered, the results given by the local sensitivity analysis are in this case coherent with those provided by the global sensitivity analysis.

Trends on SIs are similar with the non-uniform pdfs. SIs obtained during this analysis are almost equal to the ones corresponding to uniform pdfs, except for viscous characteristic length which exhibits a strong sensitivity under $500 \mathrm{~Hz}$. Moreover in this case, the coupling effects are no longer negligible. In particular, tortuosity, which had a TSI of $20 \%$ above $2000 \mathrm{~Hz}$ for absorption coefficient, is now close to $90 \%$. This was not detected by the local analysis, so even for small parameters variability, one has to take care of simplified approaches if precise parameters' impact are required. Another point that should be emphasized is the very close behavior of porosity and flow resistivity in terms of sensitivity when considering the absorption coefficient. This observation can be done for both SIs and TSIs, which are all quite small (smaller than 20\%). This indicates that when observing only this output, separation between these parameters will be difficult. In terms of identification, this is coherent with the observation by Bolton et al. [10,11], who have shown that the effects of these two variables where very close and that removing one of them (namely fixing the value of porosity) was improving the conditioning of the identification procedure.

As a conclusion, it has been shown in this part that for reduced design spaces, the local sensitivity analysis can give coherent trends as a first step. However, when the ranges of parameters are growing, coupling effects rapidly appear and

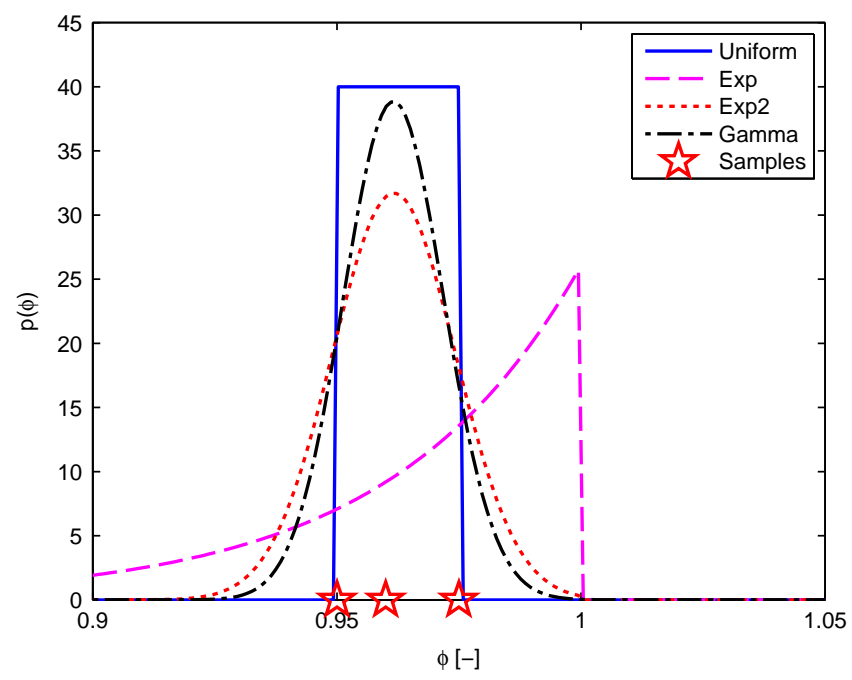

Fig. 7. Examples of pdfs for porosity. 

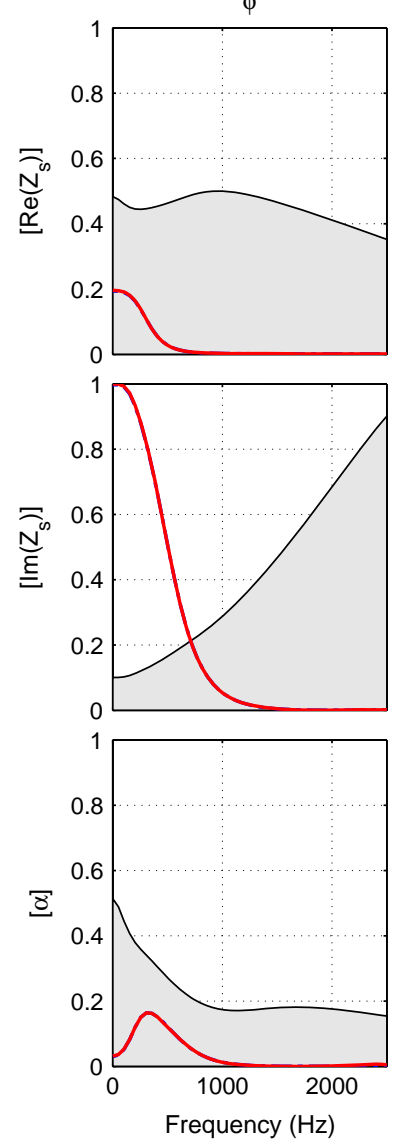
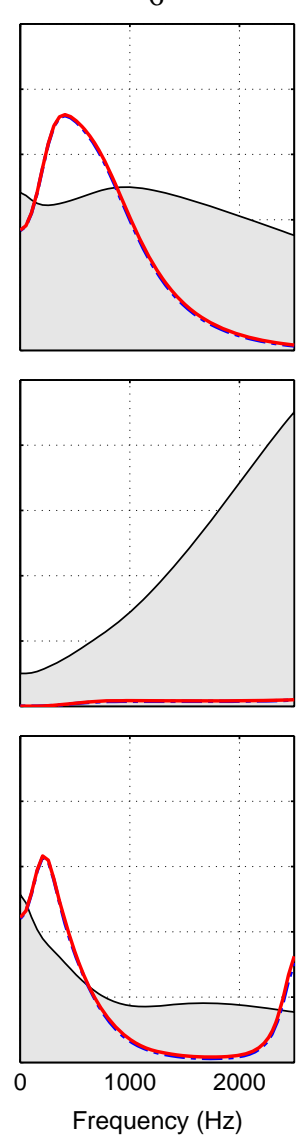

$\alpha_{\infty}$
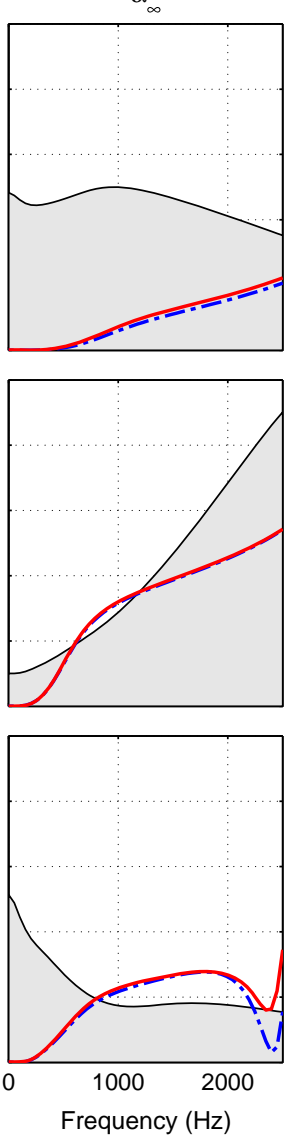

$\Lambda$
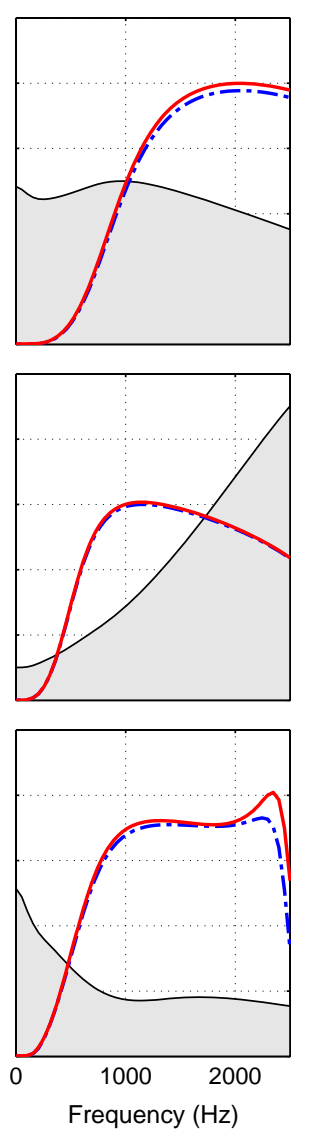

$\Lambda^{\prime}$
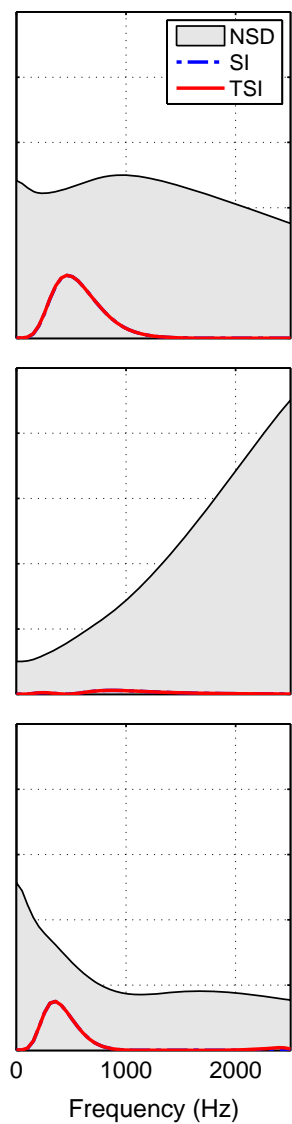

Fig. 8. Sensitivity indexes_-uniform pdfs for all variables.

the sensitivity indexes can be very badly estimated by the local approach. In particular, the choice of the pdf has an important impact on the results: switching from uniform pdfs to non-uniform pdfs implies that the minimum and maximum possible values of parameters are outside of the bounds corresponding to the uniform pdfs case, and facilitate the apparition of coupling effects. The knowledge of the physical limits associated to each parameter is then a key aspect of the problem, since they drive the results of the sensitivity analysis.

\section{Sensitivity analysis of Biot-Allard model for a wide range of materials}

In this part, a global sensitivity analysis is performed using the Biot-Allard model, for a wide range of materials. The variations of the considered parameters are given in Table 4.

The results of the sensitivity analysis are given in Fig. 10. The very interesting result here is that the SIs and TSIs obtained in this case are very close to those presented for the Champoux-Allard model (Fig. 1): the five parameters of the fluid phase have similar sensitivities in both Champoux-Allard and Biot cases. The mechanical parameters have SIs which are close to zero, but exhibit coupling effects up to 35\%. In terms of material choice, this tends to indicate that if the choice is related to either impedance or absorbing coefficient, material's characteristics must be chosen according to the fluid phase's parameters. As it will be illustrated later, mechanical parameters are becoming important when the design space is restricted to a reduced domain.

\section{Sensitivity analysis on a sample of polymer foam}

The previous analysis is repeated in this section considering a specific material, namely a polymer foam, which has been described and characterized in [9]. Uncertainties levels given in Table 5 are related to measurement, anisotropy and heterogeneity of the sample.

Fig. 11 shows the results of the sensitivity analysis when uniform pdfs are considered for each parameter. The thickness of the sample is $25 \mathrm{~mm}$. Compared to the previous analysis, the parameters ranking has been modified in a very large scale. The frequency dependency of the sensitivity index is very important. Globally, flow resistivity has the highest impact on 

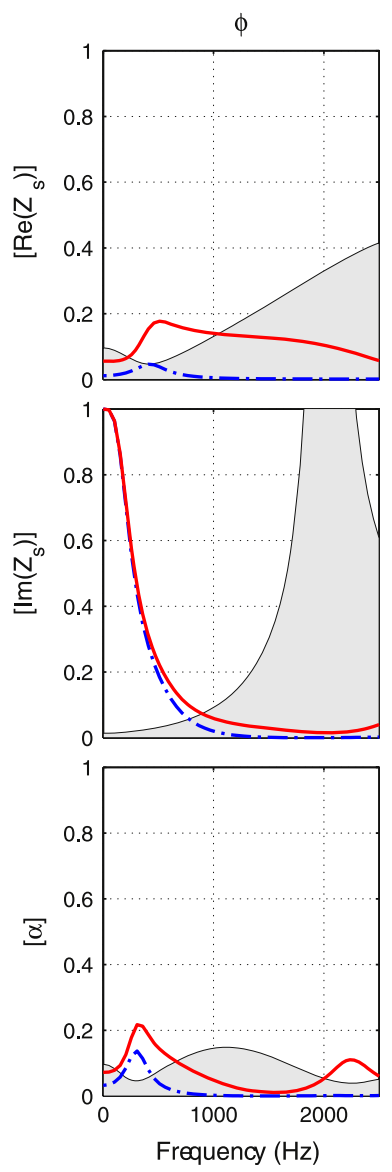

$\sigma$
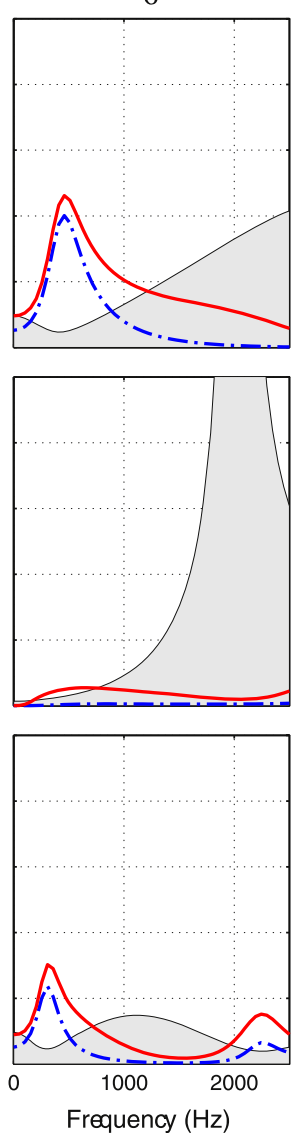

$\alpha_{\infty}$
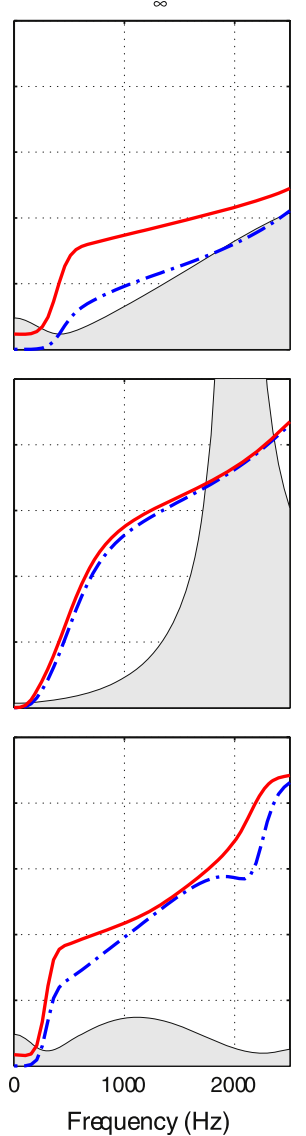

$\Lambda$
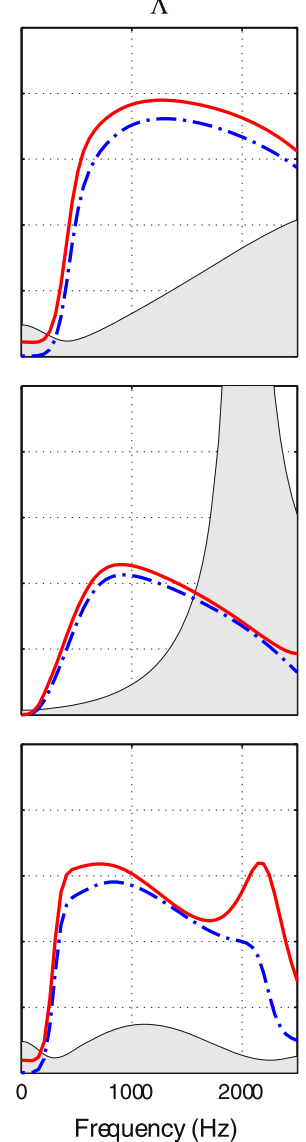

$\Lambda$,
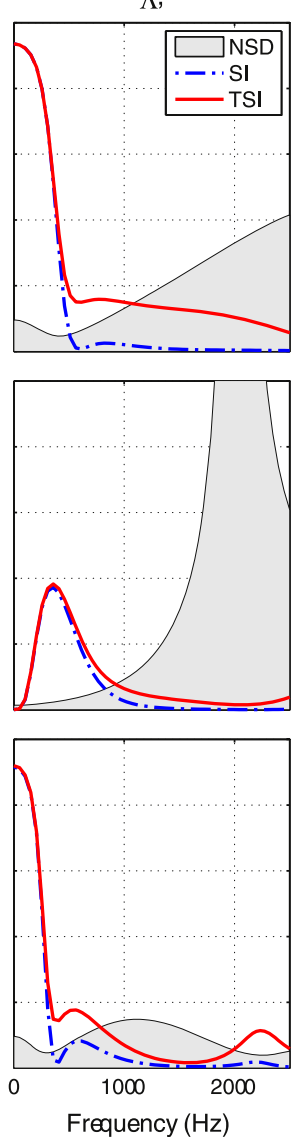

Fig. 9. Sensitivity indexes—non-uniform pdfs for all variables.

Table 4

Input parameters for Biot-Allard model (wide range of materials).

\begin{tabular}{llll}
\hline Parameter & Variable & Inferior limit & Superior limit \\
\hline Porosity & $\phi(-)$ & 0.7 & 0.99 \\
Flow resistivity & $\sigma\left(\mathrm{N} \mathrm{s} \mathrm{m}^{-4}\right)$ & 1500 & 200000 \\
Tortuosity & $\alpha_{\infty}(-)$ & 1 & 3 \\
Viscous characteristic length & $\Lambda(\mathrm{m})$ & 5 & 200 \\
Thermal characteristic length & $\Lambda^{\prime}(\mathrm{m})$ & 5 & 400 \\
Elastic modulus & $E(\mathrm{~Pa})$ & 1000 & 5000000 \\
Poisson ratio & $v(-)$ & 0 & 0.44 \\
Density & $\rho_{S}\left(\mathrm{~kg} \mathrm{~m}^{-3}\right)$ & 8 & 200 \\
Loss factor & $\eta(-)$ & 0 & 1 \\
\hline
\end{tabular}

the features, but some mechanical parameters, namely Young's modulus and Poisson ratio, are very important, in particular in zones in which mechanical effects occur. Coupling effects are almost negligible, except in these zones, which can be identified in Fig. 12, which shows the frequency evolution of the absorption coefficient for the mean values of parameters. In particular, even if the input uncertainty level is higher for $E( \pm 17 \%)$ than for $v( \pm 3 \%)$, a very large part of the global sensitivity is associated to the Poisson ratio in the frequency ranges in which mechanical resonances occur. This point illustrates the need of efficient experimental methodologies to improve identification of Poisson ratio in porous materials.

In order to understand the effect of the thickness of the sample, the absorption coefficient for a sample thickness of $47 \mathrm{~mm}$ is also given in Fig. 12.

The corresponding sensitivity analysis results are shown in Fig. 13. It can be observed that the global trend is a homothetic frequency shift: the mechanical effects have strong influence at a lower frequency, due to the higher thickness, and in the zones in which their impact is low, the flow resistivity has the highest sensitivity indexes. 


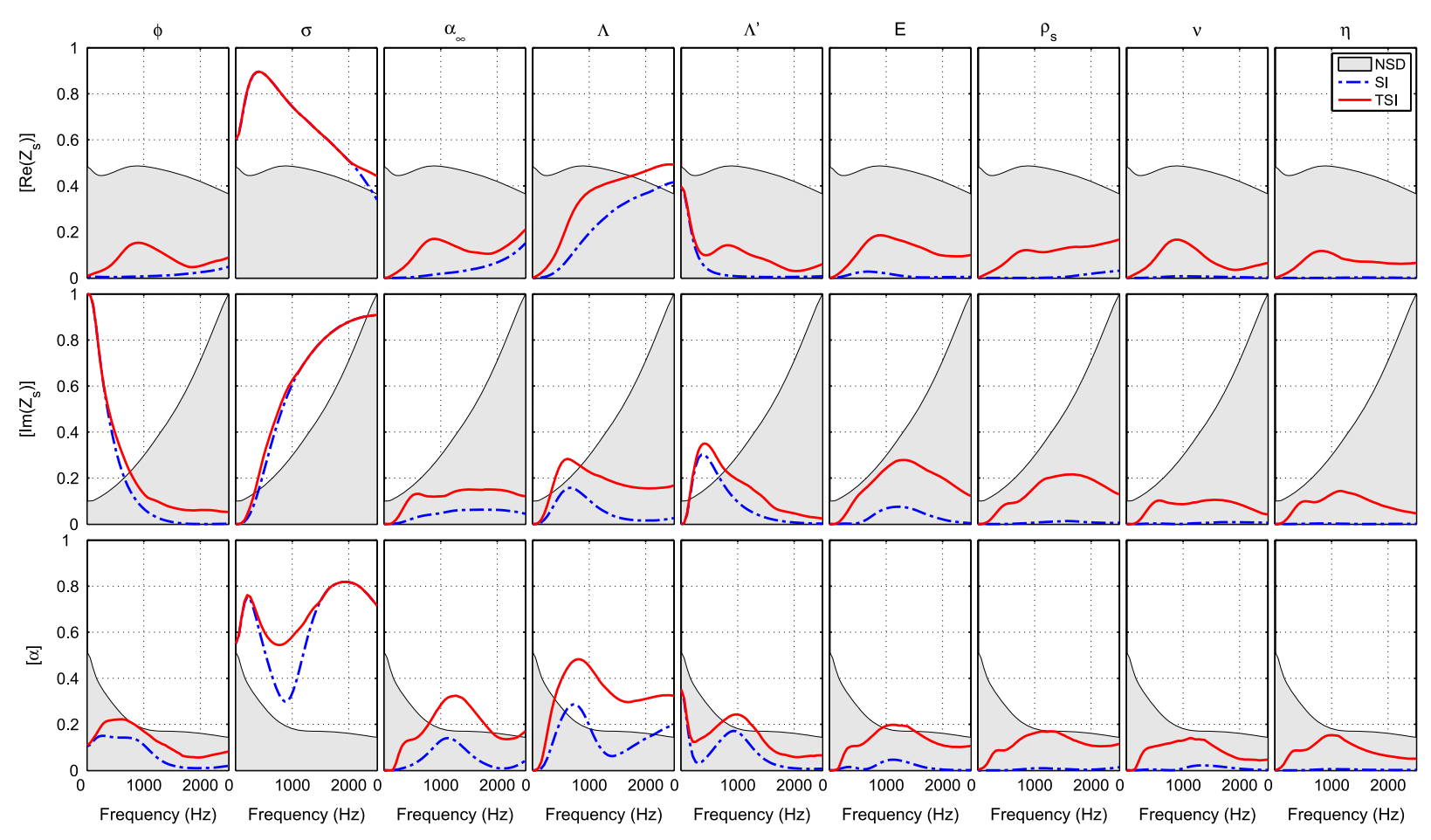

Fig. 10. Sensitivity analysis of Biot-Allard model for a wide range of materials.

Table 5

Input parameters for polymer foam [9].

\begin{tabular}{llll}
\hline Parameter & Variable & Inferior limit & Superior limit \\
\hline Porosity & $\phi(-)$ & 0.96 & 0.98 \\
Flow resistivity & $\sigma\left(\mathrm{N} \mathrm{s} \mathrm{m}^{-4}\right)$ & 119000 & 212000 \\
Tortuosity & $\alpha_{\infty}(-)$ & 1.5 & 2.1 \\
Viscous characteristic length & $\Lambda(\mathrm{m})$ & 41 & 79 \\
Thermal characteristic length & $\Lambda^{\prime}(\mathrm{m})$ & 137 & 223 \\
Elastic modulus & $E(\mathrm{~Pa})$ & 170000 & 241000 \\
Poisson ratio & $v(-)$ & 0.435 & 0.465 \\
Density & $\rho_{S}\left(\mathrm{~kg} \mathrm{~m}^{-3}\right)$ & 39.1 & 39.9 \\
Loss factor & $\eta(-)$ & 0.10 & 0.12 \\
\hline
\end{tabular}

\section{Conclusions}

Models considered for porous materials analysis are defined according to some parameters. The Champoux-Allard model (with five material parameters) and the Biot-Allard model (with nine parameters) are considered in this work for sensitivity purposes, as examples. The work presented in this paper quantifies the sensitivity of parameters for these models in the case of a $25 \mathrm{~mm}$ thick porous material sample with rigid frame, considering surface impedance and acoustic absorption indicators.

The sensitivity analysis is frequency dependent and uses two methods: Sobol and extended FAST. As expected, both sensitivity methods are in good agreement when comparing first-order and total sensitivity indexes, including coupling effects. Both techniques lead to similar results concerning the first-order and total sensitivity indexes, even if the FAST technique is faster, but provides only mean first-order and total sensitivity indexes (which are often sufficient in practical cases). The results are presented together with the normalized standard deviation to improve readability and interpretation of results.

The studies performed on porous materials illustrate the preponderant impact of flow resistivity on acoustic performances. Nevertheless, some other parameters can have a strong impact on the vibroacoustic behavior. The sensitivity of these parameters is strongly frequency dependent, since some of them can be irrelevant in a frequency band, and becoming very important for other frequency ranges. It appears that no general hierarchy of parameters for porous materials can be drawn. For the cases which have been studied, all acoustic parameters (i.e. those related to the fluid phase) have exhibited an important participation to the total sensitivity for one of the considered outputs 


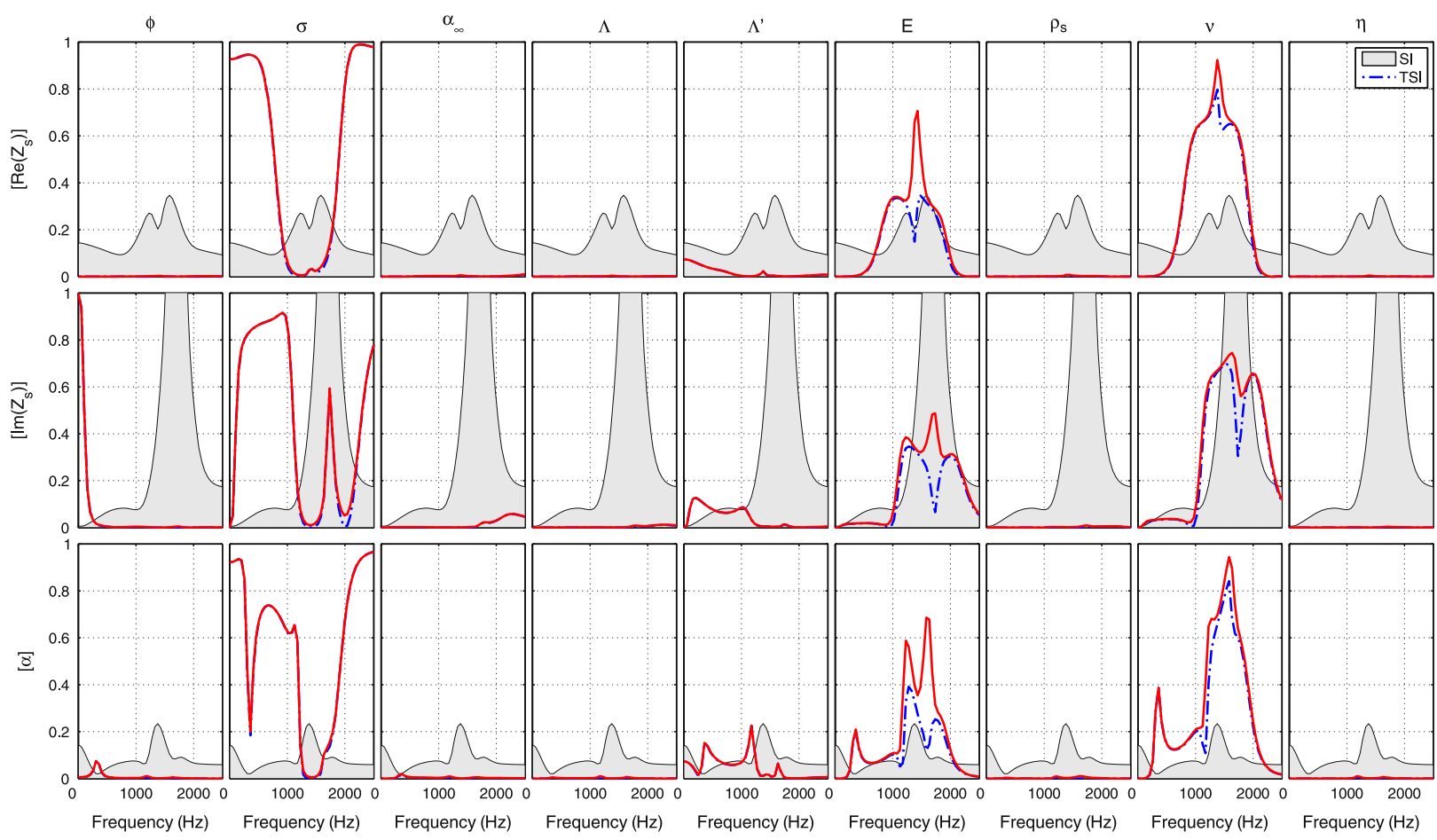

Fig. 11. Sensitivity indexes of Biot-Allard model for a polymer foam ( $25 \mathrm{~mm}$ thickness).

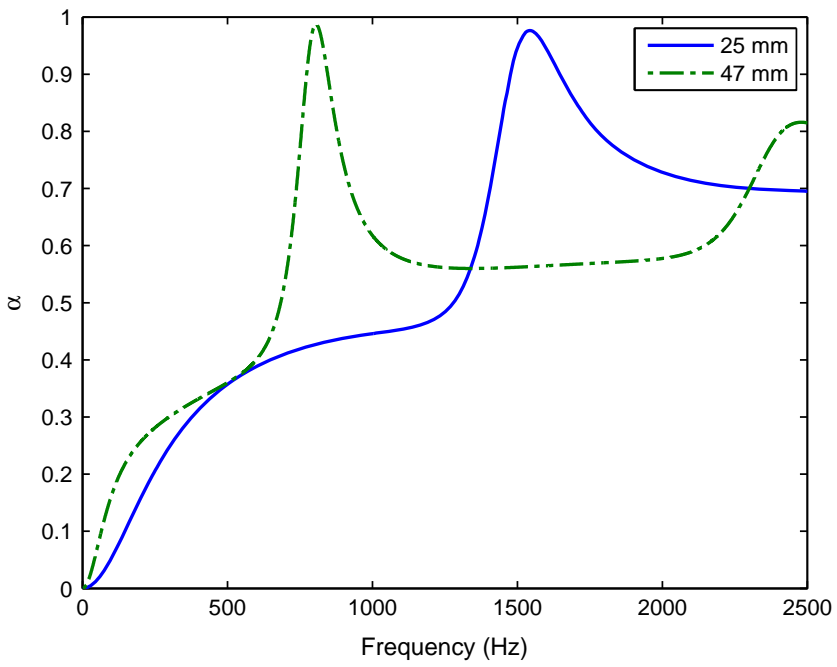

Fig. 12. Absorption coefficient for nominal values of parameters.

(real, imaginary part of impedance, absorption coefficient) for a given frequency range. Mechanical parameters are generally less influent, except in the zones in which strong couplings between fluid and solid phases appear. In these zones, elastic parameters are very important, in particular the Poisson ratio. This can lead to drastic change in the material vibroacoustic behavior even for limited fluctuation of the parameter.

One of the key aspects for the sensitivity analysis is the designer's knowledge concerning parameters variabilities: consequences in terms of hierarchy and quantification of coupling effects can strongly depend on the upper and lower bounds defined for the parameters, or by the probability density functions which are chosen.

It has been shown that global sensitivity analysis can help the designer to choose a material, since it allows one to focus on the most influent parameters of the material for the desired vibroacoustic output. It is also very useful for parameter identification purposes, since it helps the analyst to mostly devote effort on observable and influent parameters only. 


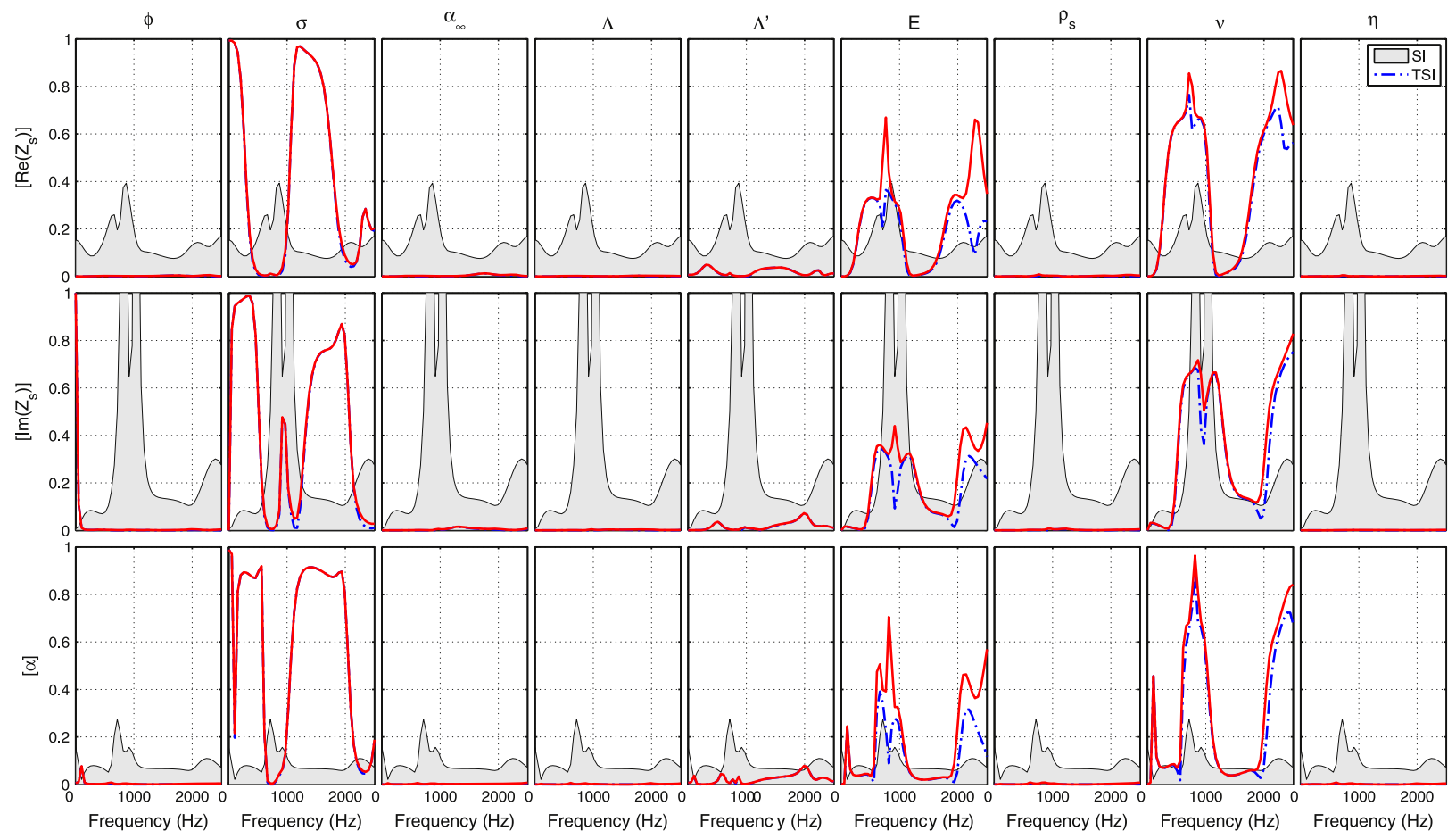

Fig. 13. Sensitivity indexes of Biot-Allard model for a polymer foam ( $47 \mathrm{~mm}$ thickness).

The first drawback of the global sensitivity analysis in this context is the large number of model evaluations which are required, due to the number of considered parameters and of the model non-linearity. Practically, this is not a drastic limitation since outputs of interest are accessible through analytical expressions, as it is the case in this paper. For more general case studies where sensitivity evaluation is expected to be time consuming, model reduction can be a solution to render this kind of analysis feasible. The second drawback in the analysis proposed here is that all material parameters are considered as independent. When the analyst has some knowledge about the relationships between the parameters, they should be taken into account in the sensitivity analysis.

\section{Appendix A. Johnson-Allard, simplified Lafarge and Champoux-Allard models}

The effective density (including viscous and inertial effects) is given by the Johnson-Allard model [7,8]:

$$
\rho_{e}(\omega)=\rho_{0}\left(\alpha_{\infty}+\frac{v_{0} \phi}{\mathrm{j} \omega q_{0}} G(\omega)\right)
$$

where $\alpha_{\infty}$ is the tortuosity, $v_{0}=\eta / \rho_{0}, \eta$ being the viscosity, $q_{0}=\eta / \sigma$ is the static viscous permeability, $\sigma$ being the flow resistivity, and:

$$
G(\omega)=\sqrt{1+\left(\frac{2 \alpha_{\infty} q_{0}}{\phi \Lambda}\right)^{2} \frac{\mathrm{j} \omega}{v_{0}}}
$$

where $\Lambda$ is the viscous characteristic length.

The bulk modulus (related to thermal effects) is given by the simplified Lafarge model [33]:

$$
K=\frac{\gamma P_{0}}{\gamma-\frac{\gamma-1}{1+\frac{v^{\prime} \phi}{\mathrm{j} \omega q_{0}^{\prime}} G^{\prime}(\omega)}},
$$

where $P_{0}$ is the static pressure, $\gamma$ is the heat capacity ratio, $v^{\prime}=v_{0} / B^{2}, B^{2}$ being the Prandtl number, $q_{0}^{\prime}$ is the static thermal permeability and

$$
G^{\prime}(\omega)=\sqrt{1+\left(\frac{2 q_{0}^{\prime}}{\phi \Lambda^{\prime}}\right)^{2} \frac{\mathrm{j} \omega}{v^{\prime}}}
$$

where $\Lambda^{\prime}$ is the thermal characteristic length. 
This model requires the knowledge of six material parameters, namely the porosity $\phi$, the flow resistivity $\sigma$, the tortuosity $\alpha_{\infty}$, the characteristic viscous and thermal lengths $\Lambda, \Lambda^{\prime}$ and the static thermal permeability $q_{0}^{\prime}$.

The Champoux-Allard model [34] is obtained by approximating the bulk modulus to the one of a material with circular cross-sectional shaped pores, yielding

$$
q_{0}^{\prime}=\frac{\phi \Lambda^{\prime 2}}{8}
$$

This model exhibits only five parameters.

\section{References}

[1] C. Zwikker, C.W. Kosten, Sound Absorbing Materials, Elsevier, 1949.

[2] M.A. Biot, Theory of propagation of elastic waves in a fluid-saturated porous solid. I. Low-frequency range, The Journal of the Acoustical Society of America 28 (1956) 168-178.

[3] M.A. Biot, Theory of propagation of elastic waves in a fluid-saturated porous solid. II. Higher frequency range, The Journal of the Acoustical Society of America 28 (2) (1956) 179-191.

[4] K. Attenborough, Acoustical characteristics of porous materials, Physics Reports 82 (3) (1982) 179-227.

[5] J.F. Allard, N. Atalla, Propagation of Sound in Porous Media: Modelling Sound Absorbing Materials, Wiley, 2009.

[6] J.F. Allard, P. Herzog, D. Lafarge, M. Tamura, Recent topics concerning the acoustics of fibrous and porous materials, Applied Acoustics 39 (1-2) (1993) $3-21$.

[7] D.L. Johnson, J. Koplik, L.M. Schwartz, New pore-size parameter characterizing transport in porous media, Physical Review Letters 57 (20) (1986) $2564-2567$.

[8] D.L. Johnson, J. Koplik, R. Dashen, Theory of dynamic permeability and tortuosity in fluid-saturated porous media, Journal of Fluid Mechanics 176 (1987) 379-402.

[9] N. Dauchez, étude vibroacoustique des matériaux poreux par éléments finis (Vibroacoustic Study of Porous Materials using Finite Elements), PhD Thesis, Université du Maine \& Université de Sherbrooke, 1999.

[10] J. Bolton, K. Hong, Inverse characterization of poro-elastic materials based on acoustical input data, 158th Meeting of the Acoustical Society of America San Antonio, TX, 2009.

[11] K.W. Hong, Acoustical Estimation of Macroscopic Properties of Poroelastic Materials, PhD Thesis, Purdue University, 2009.

[12] A. Saltelli, K. Chan, E.M. Scott, et al., Sensitivity Analysis, Vol. 475, 2000, Wiley New York, http://www.amazon.fr/Sensitivity-Analysis-A-Saltelli/dp/ $0471998923 \#$ reader_0471998923.

[13] F.O. Hoffman, J.S. Hammonds, Propagation of uncertainty in risk assessments: the need to distinguish between uncertainty due to lack of knowledge and uncertainty due to variability, Risk Analysis 14 (5) (1994) 707-712.

[14] J.C. Helton, J.D. Johnson, C.J. Sallaberry, C.B. Storlie, Survey of sampling-based methods for uncertainty and sensitivity analysis, Reliability Engineering E System Safety 91 (10-11) (2006) 1175-1209.

[15] H.C. Frey, S.R. Patil, Identification and review of sensitivity analysis methods, Risk Analysis 22 (3) (2002) 553-578.

[16] A. Saltelli, R. Bolado, An alternative way to compute Fourier amplitude sensitivity test (FAST), Computational Statistics \& Data Analysis 26 (4) (1998) $445-460$.

[17] A. Saltelli, S. Tarantola, K.P.S. Chan, A quantitative model-independent method for global sensitivity analysis of model output, Technometrics 41 (1) (1999) 39-56.

[18] A. Saltelli, T.H. Andres, T. Homma, Sensitivity analysis of model output: an investigation of new techniques, Computational Statistics E Data Analysis 15 (2) (1993) 211-238

[19] A. Saltelli, M. Ratto, S. Tarantola, F. Campolongo, Sensitivity analysis practices: strategies for model-based inference, Reliability Engineering E System Safety $91(10-11)$ (2006) 1109-1125.

[20] K. Chan, A. Saltelli, S. Tarantola, Sensitivity analysis of model output: variance-based methods make the difference, Proceedings of the 29th Conference on Winter Simulation, IEEE Computer Society, 1997, pp. 261-268.

[21] I.M. Sobol, Global sensitivity indices for nonlinear mathematical models and their Monte Carlo estimates, Mathematics and Computers in Simulation $55(1-3)(2001) 271-280$.

[22] R.I. Cukier, C.M. Fortuin, K.E. Shuler, A.G. Petschek, J.H. Schaibly, Study of the sensitivity of coupled reaction systems to uncertainties in rate coefficients. I. Theory, The Journal of Chemical Physics 59 (1973) 3873.

[23] J. Jacques, C. Lavergne, N. Devictor, Sensitivity analysis in presence of model uncertainty and correlated inputs, Reliability Engineering E System Safety $91(10-11)(2006) 1126-1134$.

[24] [link]. URL: 〈http://sensitivity-analysis.jrc.ec.europa.eu/〉.

[25] J.H. Schaibly, K.E. Shuler, Study of the sensitivity of coupled reaction systems to uncertainties in rate coefficients. II. Applications, The Journal of Chemical Physics 59 (1973) 3879.

[26] R.I. Cukier, J.H. Schaibly, K.E. Shuler, Study of the sensitivity of coupled reaction systems to uncertainties in rate coefficients. III. Analysis of the approximations, The Journal of Chemical Physics 63 (1975) 1140.

[27] M.E. Delany, E.N. Bazley, Acoustical properties of fibrous absorbent materials, Applied Acoustics 3 (2) (1970) 105-116

[28] N. Sellen, M.A. Galland, O. Hilbrunner, Identification of the characteristic parameters of porous media using active control (acoustic measurements), 8th AIAA/CEAS Aeroacoustics Conference and Exhibit, Breckenridge, CO, 2002.

[29] E.T. Jaynes, Information theory and statistical mechanics. I, Physical Review 106 (4) (1957) 620-630.

[30] C. Soize, Probabilité et modélisation des incertitudes, éléments de base et concepts fondamentaux (Probability and modelization of uncertainties, basics and fundamentals), Lecture at Université de Marne la Vallée, 2004.

[31] G.J. Hahn, S. Shapiro, Statistical Models in Engineering, John Wiley \& Sons, Hoboken, NJ, 1994.

[32] J. Kapur, Maximum-entropy Models in Science and Engineering, John Wiley \& Sons, 1989.

[33] D. Lafarge, P. Lemarinier, J.F. Allard, V. Tarnow, Dynamic compressibility of air in porous structures at audible frequencies, The Journal of the Acoustical Society of America 102 (1997) 1995.

[34] Y. Champoux, J.F. Allard, Dynamic tortuosity and bulk modulus in air-saturated porous media, Journal of Applied Physics 70 (4) (1991) 1975-1979. 